\title{
Microbiological aspects of peritonitis in patients undergoing chronic peritoneal dialysis at the dialysis unit of Sri Jayawardenapura General Hospital
}

\author{
Shalinie Perera' and C Palasuntheram² \\ (Index words: High incidence, leucocyte and neutrophil counts in dialysis effluent, causative organisms).
}

\begin{abstract}
Background Peritoneal dialysis (PD) is an established form of therapy in the management of end stage renal disease. Peritonitis is the main complication of PD.

Objectives To study the incidence and microbial aetiology of peritonitis in patients undergoing chronic PD at the dialysis unit of Sri Jayewardenapura General Hospital (SJGH); to assess the diagnostic value of the Gram's stain; and to study the relationship of the total white cell count of effluent to peritonitis.
\end{abstract}

Design A prospective. study over three months.

Setting Dialysis unit of SJGH.

Patient population The study involved 18 patients undergoing manual intermittent peritoneal dialysis (IPD), 4 patients undergoing chronic ambulatory peritoneal dialysis (CAPD), and 1 patient undergoing nocturnal intermittent peritoneal dialysis (NIPD).

Measurements Clinical presentation of patients with peritonitis; total and differential white blood cell counts of effluent samples; Gram stain and culture of the centrifuged deposit to determine microbial aetiology; incidence of peritonitis in different categories of dialysis.

Results 32 samples were examined from patients on IPD, and 17 from patients on CAPD. In IPD most episodes were due to Gram negative organisms whereas in CAPD most episodes were due to Gram positive organisms. Sensitivity of Gram's stain in relation to culture was $32.4 \%$. $98 \%$ of effluent samples had white blood cell counts of $>100 / \mathrm{ml}$ and none showed neutrophil counts of $<49 \%$.

Conclusions The incidence of IPD associated peritonitis was 11.1 episodes per patient year, and the incidence of CAPD associated peritonitis was 14 episodes per patient year. Flavobacterium spp. were the predominant organisms in IPD associated peritonitis, whereas CAPD associated peritonitis was commonly caused by coagulase negative staphylococci. Gram's stain was not useful in the initial identification of the causative agent, but the white cell and neutrophil counts were found to be sensitive indicators of peritonitis.

\section{Introduction}

PD is an established form of therapy in the management of end stage renal disease $(1,2)$.

In manual intermittent peritoneal dialysis (IPD), dialysis is carried out 2 to 3 times a week intermittently in hospital, and about 20 one litre exchanges are carried out within 24 hours. In continuous ambulatory peritoneal dialysis 2 litre exchanges are carried out continuously 3 to 4 times a day. Chronic cyclic peritoneal dialysis (CCPD) and nocturnal intermittent peritoneal dialysis (NIPD) are automated techniques of dialysis where a cycler is programmed to deliver the fluid and exchanges are done in the night.

Peritonitis is the main complication of PD $(3,4)$, and remains a cause of technique failure, hospitalisation and transfer to haemodialysis (HD), with mortality rates varying from 2 to 25\% (5). There are no previous studies in Sri Lanka on peritonitis in chronic PD systems, hence the need for the present study.

\section{Methodology}

The PD effluents processed in the study were from patients who had symptoms and signs of peritonitis or cloudy dialysate. 49 effluent samples were examined during the 3 months. A white blood cell count was performed on the uncentrifuged sample, and the centrifuged deposit was used to inoculate culture plates and make smears for the Gram's stain and Leishman's stain.

API 20E was used to identify members of the family Enterobacteriacae. Non-fermenting oxidase positive organisms such as Pseudomonas spp., Flavobacterium spp. and Gram positive organisms were identified using standard microbial identification procedures (7). The single yeast culture was identified by Gram stain and germ tube tests. Antibiotic sensitivity tests were performed using the modified Kirby Bauer technique.

Patients were considered to have peritonitis if they met at least two of the following criteria $(2,6)$.

1. Signs and symptoms of peritonitis (abdominal pain, rebound tenderness, fever, vomiting, diarthoea) 
2. A cloudy dialysate with a leucocyte count of more than $100 / \mathrm{ml}$.

3. A positive culture or a positive Gram's stain

\section{Results}

Of the 49 samples studied, 47 were from patients who had peritonitis and 2 from patients who had cloudy dialysates but did not have peritonitis. Of the 47 effluent samples, 32 were from patients on IPD and 15 from patients on CAPD. The patient on NIPD did not have peritonitis during this period.

Clinical presentation of patients with peritonitis varied. All patients with symptomatic peritonitis had diffuse abdominal pain $(80 \%)$. Abdominal tenderness was found in $48 \%$, fever in $36 \%$, vomiting in $14 \%$ and diarrhoea in $6 \%$.

Of the samples processed, except for one effluent sample which was not turbid and showed a cell count of $<100 / \mathrm{ml}$, the others were turbid and had white cell counts of $>100 / \mathrm{ml}$. $94 \%$ showed neutrophil counts of $>70 \%$, and none showed neutrophil counts of $\leq 49 \%$.

The Gram stain was positive in only 12 effluent samples giving a sensitivity of $32.4 \%$ compared to culture. The specificity and positive predictive values of Gram stain were $100 \%$, and the negative predictive value $35 \%$. Table 1 and 2 show the microbial aetiology in IPD and CAPD associated peritonitis. In this study $21.2 \%$ of cases of peritonitis were culture negative. Studies have shown that 10 to $50 \%$ of clinically infected PD effluents are culture negative $(1,4,8)$.

\section{Discussion}

Accurate diagnosis of peritonitis is an important requirement for a successful dialysis program. Symptoms and signs are an important component of the definition that aid in the diagnosis, and the frequency of different symptoms in the present study was similar to previous studies reported $(1,4)$.

Cell count and Gram stain are also important in the preliminary diagnosis of peritonitis as the clinical presentation is variable and sometimes patients are asymptomatic. A cell count indicates whether the turbidity of the dialysate is due to the presence of increased number of leucocytes. Since $98 \%$ of samples from patients with peritonitis had leucocyte counts of $>100 / \mathrm{ml}$ and none showed neutrophil counts of $\leq 49 \%$, this study indicates that a leucocyte count of $>100 / \mathrm{ml}$ and $>50 \%$ neutrophils are sensitive indicators of peritonitis.

Table 1. Microbial aetiology in IPD-associated peritonitis

Organism

Gram negative

Gram positive

Sterile

Number of episodes

Percentage
1. Flavobacterium spp
2. Acinetobacter calcoaceticus
3. Pseudomonas spp.
4. Serratia marcescens
5. Aeromonas caviae
6. Pseudomonas aeruginosa + Acinetobacter calcoaceticus

Total

7. Coagulase negative staphylococcus

8. Staphyococcus aureus

9. Enterococcus faecalis

10. Diptheroids

11. $\beta$-haemolytic streptococcus Group-C + Diptheroids

10


Table 2. Microbial aetiology in CAPDassociated peritonitis
Organism

Gram positive

Gram negative

Fungal

Total culture positive

Sterile

Coagulase negative staphylococcus

Total 4

Pseudomonas aeruginosa 2 Klebsiella spp.

Total

Candida spp.

Total
As the sensitivity of the Gram stain was only $32.4 \%$, it is not a sensitive indicator of peritonitis. In other studies reported the sensitivity of Gram stain in relation to culture varies between 10 and $50 \%(4,5,8,10)$. The Gram stain though only positive in a low percentage of cases should be performed routinely because of its simplicity and its value in making a preliminary identification of the organism in a short time to aid in the choice of antibiotics.

The organisms responsible for IPD associated peritonitis were those found in the hospital environment. Flavobacterium spp, the commonest isolate, was isolated from a sink in the dialysis unit. Flavobacterium spp have been increasingly associated with infectious complications in hospital settings, especially in immunocompromised patients (11). In a study at the National Hospital of Sri Lanka of complications of acute peritoneal dialysis, $86.7 \%$ of culture positive peritonitis were due to coliforms and $10 \%$ to Staphylococcus aureus (12).

As in previous studies most cases of peritonitis in CAPD patients in the present study were due to skin commensals, with the predominant isolate being Staphylococcus epidermidis.

The most likely portal of entry of the causative organism in IPD and CAPD associated peritonitis in our study is the lumen of the catheter. In IPD contamination occurs during multiple connections and disconnections of the PD transfer set to the catheter. We observed that there was no adherence to strict sterile conditions during this procedure. As a result the incidence of IPD associated peritonitis was 11.1 episodes per patient year. The incidence of CAPD associated peritonitis was 14 episodes per patient year. This is high compared to centres in the West where it occurs on average 1 to 2 episodes per patient year $(4,12,13)$. Poor hygienic conditions, associated with poor socioeconomic status, compared to patients in the West, as well as inadequate training in CAPD procedure, have contributed to the high incidence of CAPD associated peritonitis.

\section{Conclusions}

The incidence of peritonitis in patients undergoing chronic PD at SJGH is high in comparison to that reported from the West.

Flavobacterium spp were the commonest organisms causing peritonitis in patients undergoing IPD, while coagulase negative Staphylococcus was the commonest in CAPD associated peritonitis. Gram stain was not very useful in the initial identification of the causative agent, while the leucocyte count and neutrophil count were sensitive indicators of peritonitis

\section{Acknowledgements}

The authors thank Dr Chula Herath, nephrologist, and staff of the dialysis unit of SJGH for their co-operation.

\section{References}

1. Drukker W. Peritoneal dialysis: a historical review. In: Maker JF; ed. Replacement of renal function by dialysis. Amsterdam: Kluwer Academic Publishers, 1992: 475-514.

2. Von Gravenitz A. Microbiological aspects of peritonitis associated with continuous ambulatory peritoneal dialysis. Clinical Microbiological Reviews 1992; 5: 36-48.

3. Gokal R, Malik NP. Peritoneal dialysis. Lancet 1999; 353: 823-8.

4. Working Party of the British Society for Antimicrobial Chemotherapy. Diagnosis and management of peritonitis in ambulatory peritoneal dialysis. Lancet 1987; 1: 845-9.

5. Males BM, Walshe JJ, Garringer L, Koshiki D. Addi check filtration, Bactec and $10 \mathrm{ml}$ culture methods for recovery of microorganisms from dialysis effluent during peritonitis in CAPD. Journal of Clinical Microbiology 1987; 23: 350-3.

6. Caravaca F, Ruiz R, Dominiguez C. Risk factors for developing peritonitis caused by microorganisms of enteral origin in patients on peritoneal dialysis. Peritoneal Dialysis International 1998; 18: 41-5.

7. Cowen and Steel's Manual for the identification of medical bacteria. Barrow GI, Feltham RKA; eds. Cambridge: Cambridge University Press, 1993.

8. Gokal R. Continuous ambulatory peritoneal dialysis. In: Marker JF; eds. Replacement of renal function by dialysis. Amsterdam: Kluwer Academic Publishers, 1992: 590-615.

9. Vas SI. Microbiological diagnosis of peritonitis in patients on continuous ambulatory peritoneal dialysis. Joumal of Clinical Microbiology 1985; 21: 522-3.

10. Verbrugh Ha, Keane WF. Bacterial growth and killing in chronic ambulatory peritoneal dialysis fluids. Journal of Clinical Microbiology 1984; 20: 199-203.

11. Manfredi R, Nanneti A, Ferri Morrena, Mastroiani A, Coronado OV. Flavobacterial sp. organisms as opportunistic bacterial pathogens during advanced HIV disease. Joumal of Infection 1999; 39: 146-52.

12. Wanigasuriya K, Haykeem L, Atukorala SD, Perera J, Sheriff R. Acute peritoneal dialysis in the National Hospital of Sri Lanka - a study of complications. Journal of the Ceylon College of Physicians 1996; 29: 23-6.

13. Keane WF, Everette ED, Golper A, Gokal R, Vas. S. Peritoneal dialysis related peritonitis: treatment recommendations. Peritoneal Dialysis Intemational 1993; 13: 2-16. 